\title{
Morfologia da copa para avaliar o espaço vital de quatro espécies nativas da Amazônia
}

\author{
Helio Tonini(1) e Marcelo Francia Arco-Verde(1) \\ (1)Embrapa Roraima, Caixa Postal 132, CEP 69301-970 Boa Vista, RR. E-mail: helio@cpafrr.embrapa.br, arcoverd@cpafrr.embrapa.br
}

\begin{abstract}
Resumo - O objetivo deste trabalho foi o de definir diretrizes para o desbaste de quatro espécies nativas: a castanha-do-pará ou castanheira-do-brasil (Bertholletia excelsa), a andiroba (Carapa guianensis), o ipê-roxo (Tabebuia avellanedae) e o jatobá (Hymenaea courbaril), plantadas em plantios homogêneos no Estado de Roraima. Foi feita a análise das relações entre os principais índices morfométricos da copa e o crescimento em diâmetro e altura; para isso foram medidos o diâmetro à altura do peito, altura total, altura de inserção e diâmetro da copa de 87 árvores. Na análise dos parâmetros de copa, a castanheira-do-brasil demonstrou superioridade em diâmetro e área, o que indica que essa espécie necessita de um maior espaço vital e maiores espaçamentos iniciais; no entanto, sua copa foi menos eficiente em manter um mesmo incremento médio anual em diâmetro.

Termos para indexação: Bertholletia excelsa, Carapa guianensis, Tabebuia avellanedae, Hymenaea courbaril, diâmetro da copa, desbastes.
\end{abstract}

\section{Crown morphology to evaluate the growing space of four Amazon native species}

\begin{abstract}
This work aimed at defining guidelines for thinning of four native species in pure stands, in Roraima State: Brazil nut tree (Bertholletia excelsa), Andiroba (Carapa guianensis), Ipê-roxo (Tabebuia avellanedae), and Jatobá (Hymenea courbaril). Analyses were made on the relations between main crown morphometric index, and diameter and height growth of these species. Eighty-seven trees were measured, and data taken are: diameter at breast height, total height, crown insertion height and crown diameter. Crown parameters analysis showed diameter and crown area superiority of Brazil nut, which indicates its need of a proper initial spacing and bigger growing space. However, Brazil nut crown was less efficient to maintain the same mean annual diameter increment.

Index terms: Bertholletia excelsa, Carapa guianensis, Tabebuia avellanedae, Hymenaea courbaril, crown diameter, thinning.
\end{abstract}

\section{Introdução}

A fonte de energia de uma árvore é a luz do sol, que é transformada pelo processo de fotossíntese em energia química, e a copa é o órgão responsável por esse processo, por isso as variáveis como superfície, diâmetro e comprimento da copa estão diretamente relacionadas com o crescimento e a produção de uma árvore. Essas variáveis são modificadas pela concorrência; a luz e o vento são os principais fatores responsáveis por essas modificações; quanto mais denso o povoamento, menos luz atinge as camadas mais baixas do dossel, o que faz com que os galhos nessas posições morram (Nuto, 2001).

Segundo Wadsworth (2000), a produtividade por unidade de área relaciona-se com a forma da copa. Copas de vários níveis, com um tronco central, produzem mais por unidade de área do que copas amplas, hemisféricas, com muitos galhos e um só nível.

Autores como Burger (1939) e Assmann (1961) foram os pioneiros na condução de estudos sobre as formas, dimensões e as relações morfométricas das copas das árvores. No Brasil, são poucos os estudos que procuram relacionar variáveis morfométricas com variáveis de fácil obtenção, como o diâmetro à altura do peito (DAP) e a altura (h). Entre os trabalhos destacam-se os de Durlo \& Denardi (1998), Spathelf et al. (2000), Durlo (2001), Nutto (2001) e Nutto et al. (2001).

Durlo \& Denardi (1998) apresentaram definições a respeito das principais variáveis morfométricas. A medição dessas variáveis, principalmente a área de copa, consome muito tempo, e é necessário utilizar diâmetro à altura do peito (DAP) e a altura (h), como variáveis independentes em equações de regressão, por serem de fácil obtenção. 
Segundo Schneider (1993), vários ensaios mostram que existe alta correlação entre o diâmetro do fuste (DAP) e o diâmetro da copa (DC). A qualidade do sítio influencia nessa correlação; quanto melhor a qualidade do sítio menor é a correlação.

O tamanho e a forma da copa influem em sua produtividade. As árvores do dossel superior têm copas horizontais, pouco densas; as do estrato inferior têm copas verticais e profundas (Wadsworth, 2000).

Segundo Dawkins (1959), citado por Wadsworth (2000), para espécies intolerantes à sombra, a razão DC/DAP não diminui à medida que a espécie alcança a maturidade, mas diminui para as tolerantes. $\mathrm{O}$ autor concluiu que a alta razão DC/DAP, necessária para o crescimento rápido de espécies tropicais, requer um bom crescimento inicial da copa e, portanto, ausência de supressão.

A relação DC/DAP expressa quantas vezes o DC é maior que o DAP. Quando se prevê o manejo de um povoamento, não pela sua idade, mas pelo diâmetro atingido por seus componentes, o número máximo de árvores por unidade de área, à medida que as árvores forem crescendo, pode ser calculado por esse índice. Essa relação pode então ser utilizada como indicador de desbaste e pode-se determinar, a qualquer tempo, o espaço a ser liberado ao redor de uma árvore selecionada, para que ela cresça sem concorrência. A relação entre o DC e o DAP, elevada ao quadrado, corresponde ao índice de espaço vital. Quanto menor for esse índice, em média no povoamento, tanto maior será a sua área basal e, provavelmente, também o seu volume por hectare (Durlo \& Denardi, 1998).

Este trabalho foi realizado com o objetivo de definir diretrizes para o desbaste de quatro espécies nativas da Amazônia, plantadas em povoamentos homogêneos, a partir da verificação das relações entre os principais índices morfométricos da copa e o crescimento em diâmetro e altura de Carapa guianensis, Bertholletia excelsa, Tabebuia avellanedae e Hymenaea courbaril.

\section{Material e Métodos}

A partir de 1995 foi iniciado, pela Embrapa Roraima, o plantio de uma coleção de espécies arbóreas nativas e exóticas (arboretos), com a finalidade de avaliar, preliminarmente, espécies potenciais para sistemas agroflorestais e programas de reposição florestal.
A área de estudos localiza-se no Campo Experimental Confiança, no município de Cantá, distante cerca de $90 \mathrm{~km}$ de Boa Vista. O clima da região é classificado como do tipo Ami (Köppen), com precipitação média de $1.900 \mathrm{~mm}$; a época chuvosa ocorre no período de abril a setembro, e o mês de junho é o mais chuvoso, com cerca de $19 \%$ da precipitação total anual.

Os dados para a realização deste estudo são oriundos de 87 árvores: 19 de Carapa guianensis (andiroba), 21 de Bertholletia excelsa (castanheira-do-brasil), 22 de Tabebuia avellanedae (ipê-roxo) e 25 de Hymenaea courbaril (jatobá). As árvores foram plantadas em parcelas retangulares de $180 \mathrm{~m}^{2}$, em espaçamento de 2,5x2 m, localizadas próximas, de forma a não existirem grandes variações de sítios, com 7 anos de idade no momento da análise. Os plantios foram feitos por coveamento direto, com a aplicação de $60 \mathrm{~g}$ de fósforo na cova. Em cada parcela, foram medidos: o diâmetro à altura do peito (DAP), a altura total (h), a altura de inserção da copa (IC) e o diâmetro da copa (DC), de todas as árvores. O diâmetro da copa foi obtido pela medição de oito raios, em direções fixas, com o auxílio de uma régua, estacas e trena.

Foram utilizadas, como variáveis morfométricas: altura de inserção da copa (IC), diâmetro da copa (DC), proporção de copa (PC), grau de esbeltez (GE), índice de saliência (IS), índice de abrangência (IA) e formal de copa (FC), conforme Burger (1939).

A proporção de copa foi calculada pela expressão $\mathrm{PC}=\frac{1}{\mathrm{~h}} \times 100$, em que $\mathrm{l}$ é o comprimento da copa em metros, obtido diminuindo-se a altura total (h) da altura de inserção de copa (IC). O grau de esbeltez foi calculado por $\mathrm{GE}=\frac{\mathrm{h}}{\mathrm{DAP}}$, em que he é a altura total em metros; DAP é o diâmetro tomado a 1,30 m, em cm. O índice de saliência foi calculado por Is $=\frac{\mathrm{DC}}{\mathrm{DAP}}$, em que DC é o diâmetro de copa em metros; DAP é o diâmetro tomado a 1,30 m em metros. O índice de abrangência foi calculado por IA $=\frac{\mathrm{DC}}{\mathrm{h}}$, em que DC é o diâmetro da copa em metros; h é a altura total em metros. O formal de copa foi obtido por $\mathrm{FC}=\frac{\mathrm{DC}}{\mathrm{l}}$, em que DC é o diâmetro da copa em metros; l é o comprimento da copa em metros.

As variáveis morfométricas da copa são consideradas de difícil obtenção, por isso é necessário o desenvolvimento de equações que as estimem, em função de variáveis de fácil obtenção, como o diâmetro à altura do 
peito e a altura. Para verificar as correlações existentes, entre as variáveis morfométricas e o diâmetro e a altura, utilizou-se o coeficiente de correlação de Pearson.

As equações para estimar o diâmetro da copa foram obtidas por análise de regressão, com a utilização do procedimento estatístico 'stepwise', em que a variável independente potencial foi selecionada para um nível de 5\% de erro. Para verificar a eficiência das equações, foram analisadas as estatísticas do coeficiente de determinação, erro padrão de estimativa, valor e significância de F para o modelo, valor e significância de t para os coeficientes e a distribuição dos resíduos.

\section{Resultados e Discussão}

Observa-se superioridade da castanheira-do-brasil, nos parâmetros de povoamento, diâmetro à altura do peito, altura dominante e altura média, o que indica maior crescimento e, conseqüentemente, maior produtividade (Tabela 1). Os valores superiores para o diâmetro e a área de copa da castanheira-do-brasil indicam que essa espécie desenvolve copas maiores e mais vigorosas, por isso necessita de maior espaço vital e exige maiores espaçamentos iniciais do que a andiroba, o ipêroxo e o jatobá. Na comparação com as demais espécies, os maiores valores de proporção de copa (PC) indicam que a castanheira-do-brasil apresenta copas mais profundas.

A razão entre DC e DAP indicou que as copas da castanheira são cerca de 50 vezes maiores que o DAP, contra 14,7 da andiroba, 32,3 do ipê-roxo e 33,1 do jatobá. Isto significa que o espaço liberado em torno de uma determinada árvore deve ser maior para a castanheira do que para as demais espécies estudadas.

Os valores encontrados neste estudo são bem maiores do que os observados por Dawkins (1963), citado por Wadsworth (2000), ao afirmar que em florestas na- turais tropicais a razão DC/DAP varia entre 14 e 28 . Segundo o autor, espécies de maior crescimento volumétrico apresentam valores entre 15 e 20; valores maiores do que 25 caracterizam espécies de baixo crescimento. Para Catinot (1974), razões grandes (acima de 40) estão relacionadas a espécies de baixa produção volumétrica na África.

A andiroba apresentou um maior grau de esbeltez, o que significa que possui forma mais alongada, em relação às demais espécies. No entanto, nenhuma espécie apresentou relação $\mathrm{h} / \mathrm{d}$ superior a 1 . Uma relação $\mathrm{h} / \mathrm{d}$ superior a 1 pode indicar a necessidade de desbaste, pois o crescimento em diâmetro é reduzido em relação à altura. A análise do formal de copa (DC/l), mostrou valores médios maiores para a castanheira-do-brasil, o que indica que a espécie possui copas mais profundas e com maiores diâmetros.

As correlações positivas do diâmetro e comprimento da copa com o DAP e a altura indicam que os mesmos crescem à medida que aumenta o DAP e a altura das árvores, em todas as espécies, com exceção da andiroba cujo DC não apresentou correlações significativas (Tabela 2)

As correlações negativas do formal de copa, índice de esbeltez e índice de saliência com o DAP e a altura indicam que os mesmos tendem a diminuir com o aumento da altura e o diâmetro das árvores; essa tendência de diminuição também foi observada por Durlo (2001), para Cabralea canjerana, em florestas naturais do Rio Grande do Sul.

A diminuição do índice de esbeltez, com o aumento da altura das árvores, indica que as mesmas estão crescendo mais em diâmetro do que em altura. O sinal negativo apresentado pelo coeficiente de correlação de Pearson, para o índice de saliência, indica que, de forma geral, o DC incrementa menos do que o DAP.

O índice de abrangência tende a diminuir com a altura, porque o aumento na altura não é acompanhado, pro-

Tabela 1. Parâmetros morfométricos e de povoamento de andiroba (Carapa guianensis), castanheira-do-brasil (Bertholletia excelsa), ipê-roxo (Tabebuia avellanedae) e jatobá (Hymenaea courbaril), aos sete anos de idade ${ }^{(1)}$.

\begin{tabular}{|c|c|c|c|c|c|c|c|c|c|c|c|c|}
\hline \multirow[t]{2}{*}{ Espécie } & \multicolumn{4}{|c|}{ Parâmetros de povoamento } & \multicolumn{8}{|c|}{ Parâmetros morfométricos } \\
\hline & $\mathrm{N}$ & $\mathrm{d}$ & $\mathrm{h}_{100}$ & $\mathrm{~h}$ & IC & $\mathrm{DC}$ & $\mathrm{AC}$ & PC & IS & GE & IA & $\mathrm{FC}$ \\
\hline Andiroba & 1.055 & 10,5 & 10,7 & 9,2 & 3,43 & 1,5 & 1,86 & 61,7 & 14,7 & 0,88 & 0,17 & 0,30 \\
\hline Castanheira-do-brasil & 1.167 & 13,5 & 13,9 & 11,3 & 2,93 & 6,6 & 35,60 & 72,7 & 50,3 & 0,84 & 0,61 & 0,86 \\
\hline Ipê-roxo & 1.222 & 10,7 & 10,2 & 8,3 & 3,40 & 3,5 & 10,40 & 58,4 & 32,3 & 0,79 & 0,41 & 0,73 \\
\hline Jatobá & 1.389 & 8,6 & 7,1 & 6,0 & 2,82 & 2,8 & 6,60 & 60,8 & 33,1 & 0,71 & 0,47 & 0,79 \\
\hline
\end{tabular}

(1)N: número de árvores por hectare; d: diâmetro à altura do peito; $\mathrm{h}_{100}$ : altura dominante (m); h: altura média (m); IC: altura de inserção da copa (m); DC: diâmetro de copa (m); AC: área de copa $\left(\mathrm{m}^{2}\right)$; PC: proporção de copa; IS: índice de saliência; GE: grau de esbeltez; IA: índice de abrangência; FC: formal de copa. 
porcionalmente, pelo aumento do DC, ou seja, o crescimento em altura ainda é maior que o crescimento do DC. No caso do ipê-roxo, a correlação, apesar de não significativa, tem sinal positivo, o que pode ser explicado pelas menores variações em altura do que em diâmetro e, uma vez que as árvores mais grossas possuem maiores diâmetros de copa, esse índice aumenta.

O incremento médio anual, em área basal, dividido pela área da copa da árvore média, pode determinar a eficiência da copa, o que descreve a eficiência de certas árvores ou espécies em utilizar um determinado espaço disponível de crescimento (Sterba, 2001). Utilizando-se valores obtidos para a árvore média de cada espécie, a eficiência da copa foi maior para a andiroba, seguida pelo ipê-roxo, jatobá e castanheira, com valores de $0,46 \%, 0,14 \%, 0,08 \%$ e $0,02 \%$, respectivamente. Isto significa que a castanheira-do-brasil, apesar de apresentar maiores dimensões, possui baixa eficiência de crescimento, por possuir copas amplas, com galhos grossos que ocupam grandes espaços no terreno.

As equações de regressão para a andiroba, castanheira-do-brasil, ipê-roxo e jatobá, obtidas com o proce-

Tabela 2. Correlação entre as variáveis morfométricas e o diâmetro e a altura das espécies estudadas ${ }^{(1)}$.

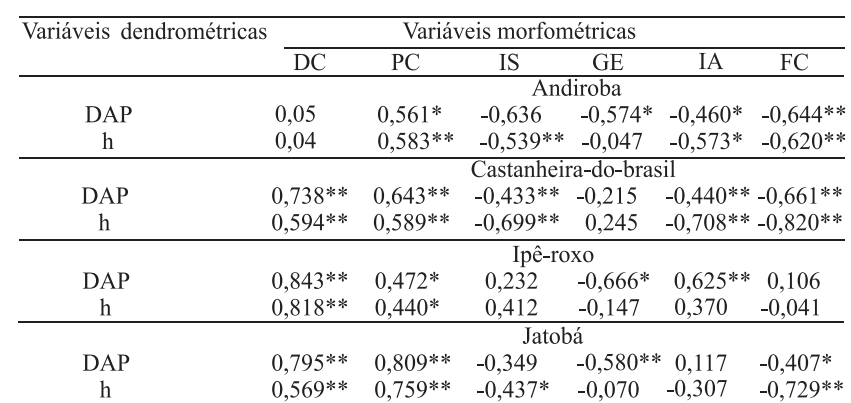

${ }^{(1) D A P: ~ d i a ̂ m e t r o ~ a ̀ ~ a l t u r a ~ d o ~ p e i t o ; ~ h: ~ a l t u r a ~ m e ́ d i a ; ~ D C: ~ d i a ̂ m e t r o ~ d e ~ c o p a ~}$ (m); PC: proporção de copa em porcentagem; IS: índice de saliência; GE: índice de esbeltez; IA: índice de abrangência; FC: formal de copa. * e **Significativo a $5 \%$ e a $1 \%$ de probabilidade, respectivamente. dimento 'stepwise', bem como o seu ajuste, são apresentadas na Tabela 3. A Figura 1 mostra o comportamento do diâmetro da copa em função do DAP, para as espécies analisadas.

Pode-se observar na Tabela 3 os baixos valores de $\mathrm{R}^{2}$ e F, para a equação utilizada para estimar o DC, em função do DAP, para a andiroba. Esses baixos valores devem-se ao fato de as copas da andiroba manteremse pequenas; quase não variam com o aumento na dimensão da árvore, como pode ser observado na Figura 1. As demais espécies apresentam uma tendência linear de aumento do DC com o DAP. Esse tipo de comportamento tem sido o mais comum, tendo sido mencionado por autores como Schneider (1993) e Nutto (2001).

O conhecimento da relação entre o DC e o DAP possibilita o cálculo do número máximo de árvores por hectare, quando um certo diâmetro ou uma certa distribuição de diâmetros é desejada. Assumindo-se que as copas tenham a forma circular, a área de copa e o número de árvores para a castanheira-do-brasil foram calculados pelas seguintes equações:

$$
\mathrm{DC}=\mathrm{EXP}^{(0,3958+0,5724 \ln \mathrm{DAP})}, \mathrm{AC}=\frac{\pi \cdot \mathrm{DC}}{4}, \mathrm{~N}=\frac{10000}{\mathrm{AC}}
$$

em que DC é o diâmetro de copa (m); DAP é o diâmetro à altura do peito; $\mathrm{AC}$ é a área de copa $\left(\mathrm{m}^{2}\right) ; \mathrm{N}$ é o número de árvores por hectare.

Utilizando-se essas equações estimou-se, o número de árvores a permanecer no povoamento, para atingir um diâmetro objetivo de 14 a $18 \mathrm{~cm}$ para a castanheirado-brasil, e de 10 a $14 \mathrm{~cm}$ para o ipê-roxo e o jatobá. Esses valores correspondem aos maiores diâmetros observados em árvores de sete anos de idade (Tabela 4).

Observa-se que para atingir um diâmetro médio de $18 \mathrm{~cm}$, incremento médio anual em diâmetro de 2,6 cm aos sete anos, deveriam permanecer no máximo

Tabela 3. Ajuste das equações obtidas com o procedimento 'stepwise’, para estimar o diâmetro da copa da andiroba (Carapa guianensis), castanheira-do-brasil (Bertholletia excelsa), ipê-roxo (Tabebuia avellanedae) e jatobá (Hymenaea courbaril), utilizando-se o diâmetro à altura do peito como variável independente $\mathrm{e}^{(1)}$.

\begin{tabular}{|c|c|c|c|c|c|c|c|c|}
\hline \multirow[t]{2}{*}{ Espécie } & \multicolumn{4}{|c|}{ Coeficientes } & \multicolumn{4}{|c|}{ Ajuste } \\
\hline & $\mathrm{b}_{0}$ & $\mathrm{~b}_{1}$ & $\mathrm{~b}_{2}$ & $b_{3}$ & $\mathrm{R}^{2}$ & Syx & $\mathrm{F}$ & prob $>\mathrm{F}$ \\
\hline Andiroba & $-30,3920$ & $-21207,7$ & 3990,09 & 1,593 & 0,15 & 0,14 & 2,40 & 0,200 \\
\hline Castanheira-do-brasil & 0,3958 & 0,5724 & & & 0,61 & 0,13 & 30,13 & 0,001 \\
\hline Ipê-roxo & $-0,1880$ & 0,2510 & & & 0,80 & 0,14 & 79,90 & 0,001 \\
\hline Jatobá & 0,1690 & 0,0970 & & & 0,61 & 0,14 & 45,90 & 0,001 \\
\hline
\end{tabular}

${ }^{(1)} b_{0}, b_{1}, b_{2}$ e $b_{3}$ : coeficientes; $R^{2}$ : coeficiente de determinação; Syx: erro padrão de estimativa; F: valor de $F$ calculado; prob>F: significância para o valor de F; equações para estimar o diâmetro da copa: $\ln \mathrm{DC}=\mathrm{b}_{0}+\mathrm{b}_{1} \frac{1}{\mathrm{~d}^{3}}+\mathrm{b}_{2} \frac{1}{\mathrm{~d}^{2}}+\mathrm{b}_{3} \mathrm{~d}$ (andiroba); $\ln \mathrm{DC}=\mathrm{b}_{0}+\mathrm{b}_{1} \ln \mathrm{d}$ (castanheira-do-brasil); $\ln \mathrm{DC}=\mathrm{b}_{0}+\mathrm{b}_{1} \ln ^{2} \mathrm{~d}$ (ipê roxo); $\ln \mathrm{DC}=\mathrm{b}_{0}+\mathrm{b}_{1} \mathrm{~d}$ (jatobá). 
211 árvores ha ${ }^{-1}$, o que significa que, nesse povoamento, deveriam ser removidas 956 árvores ha ${ }^{-1}$, ou seja, $82 \%$ das árvores. Esse resultado concorda com as afirmações de Yared et al. (1993), ao afirmarem que o número de árvores a permanecer no corte final, para uma idade de rotação de 30 a 40 anos, deve ser próximo de 100 árvores ha ${ }^{-1}$. No entanto, segundo esses autores, a densidade inicial de plantio para a produção de madeira deve estar entre $16 \mathrm{~m}^{2}$ e $25 \mathrm{~m}^{2}$ por planta, valor mais baixo do que o encontrado neste estudo.
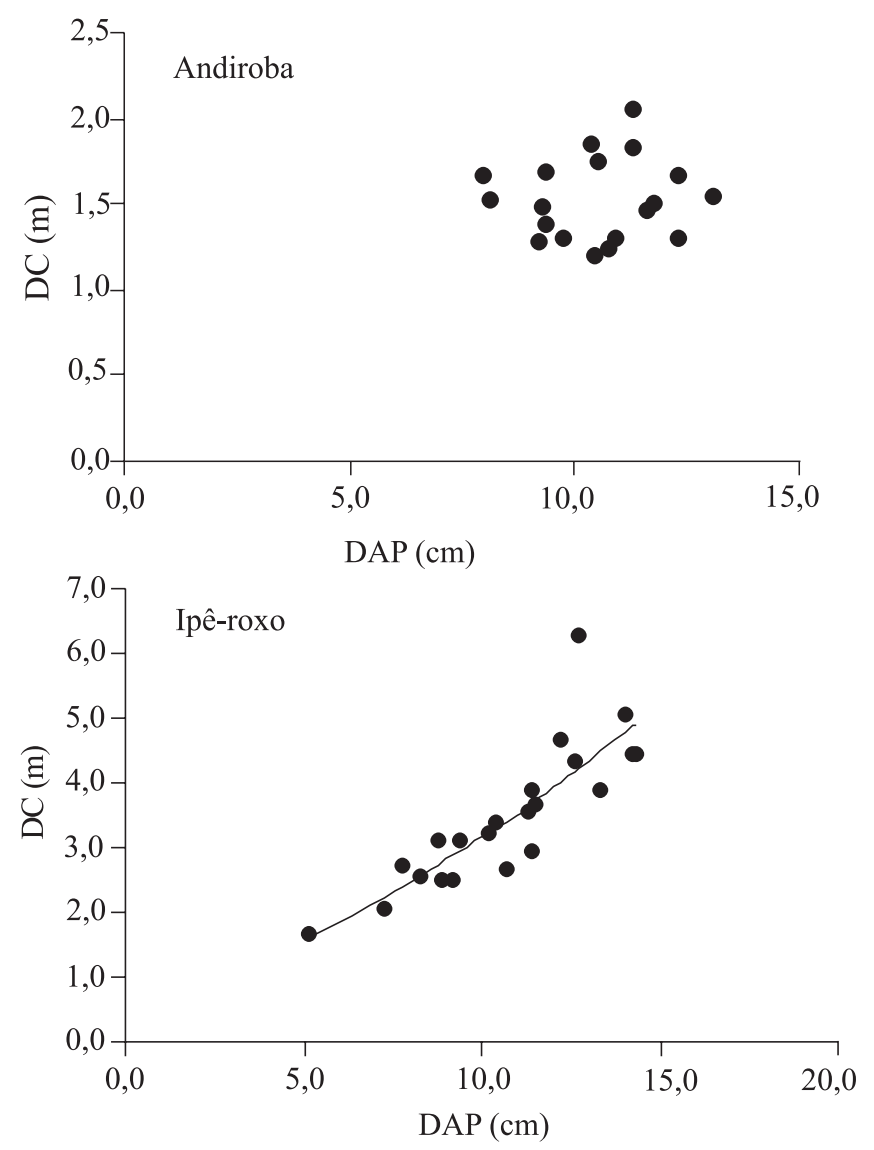

Pode-se observar que para atingir um diâmetro objetivo de $14 \mathrm{~cm}$, incremento médio anual de $2 \mathrm{~cm}$, o ipêroxo apresenta uma área de copa de $17,79 \mathrm{~m}^{2}$, e o número de árvores máximo por hectare é de 562, o que significa uma redução de $54 \%$ em relação ao número de árvores atual (Tabela 4).

Para um mesmo diâmetro e incremento médio anual, o jatobá apresenta uma área de copa de $16,65 \mathrm{~m}^{2}$, e o máximo número de árvores, por hectare, a permanecer é de 601, o que significa uma redução de $43 \%$ em relação ao povoamento atual.
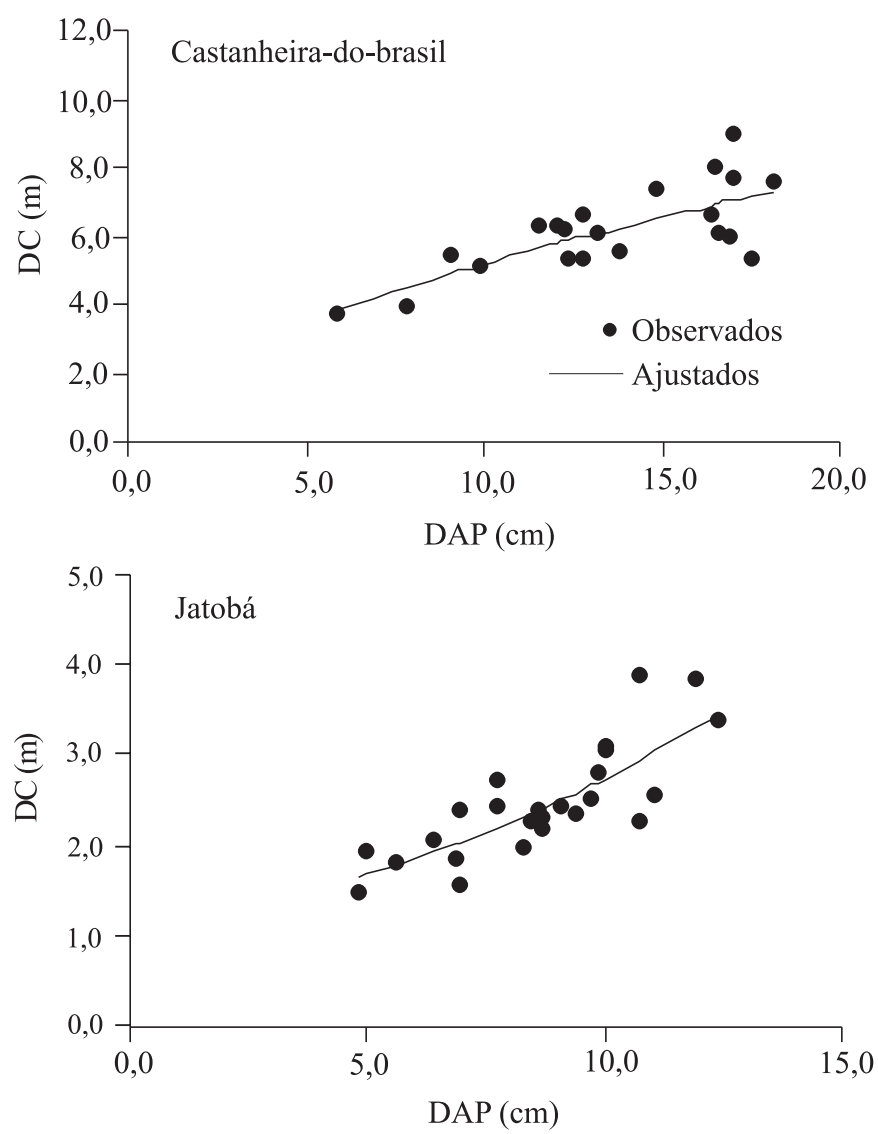

Figura 1. Relação do diâmetro de copa (DC) com o diâmetro à altura do peito (DAP), nas espécies estudadas.

Tabela 4. Espaço vital para a castanheira-do-brasil (Bertholletia excelsa), ipê-roxo (Tabebuia avellanedae) e jatobá (Hymenaea courbaril), para um determinado diâmetro objetivo aos sete anos de idade.

\begin{tabular}{|c|c|c|c|c|c|c|c|c|c|}
\hline \multirow{2}{*}{$\begin{array}{l}\text { Diâmetro } \\
\text { objetivo }(\mathrm{cm})\end{array}$} & \multicolumn{3}{|c|}{ Diâmetro da copa $(\mathrm{cm})$} & \multicolumn{3}{|c|}{ Área da copa $\left(\mathrm{m}^{2}\right)$} & \multicolumn{3}{|c|}{ Número de árvores ha ${ }^{-1}$} \\
\hline & Castanheira-do-brasil & Ipê-roxo & Jatobá & Castanheira-do-brasil & Ipê-roxo & Jatobá & Castanheira-do-brasil & Ipê-roxo & Jatobá \\
\hline 10 & & 3,14 & 3,1 & & 7,72 & 7,66 & & 1.295 & 1.305 \\
\hline 12 & 6,16 & 3,90 & 3,8 & 29,82 & 11,96 & 11,29 & 335 & 836 & 885 \\
\hline 14 & 6,73 & 4,76 & 4,6 & 35,57 & 17,79 & 16,65 & 281 & 562 & 601 \\
\hline 16 & 7,27 & & & 41,45 & & & 241 & & \\
\hline 18 & 7,77 & & & 47,44 & & & 211 & & \\
\hline
\end{tabular}


Esta análise permite comprovar a baixa eficiência das copas da castanheira-do-brasil em manter um incremento médio anual em diâmetro de $2 \mathrm{~cm}$, pois para isso necessita de uma área de copa de 35,57 $\mathrm{m}^{2}$, contra $17,79 \mathrm{~m}^{2}$ do ipê-roxo e 16,65 m² do jatobá.

\section{Conclusões}

1. Pode-se afirmar que existem relações significativas entre diversos índices morfométricos e o diâmetro e a altura, das árvores das espécies estudadas; o diâmetro e o comprimento da copa crescem, à medida que aumentam o DAP e a altura (com exceção da andiroba); o formal de copa, o índice de esbeltez e o índice de saliência tendem a diminuir.

2. A análise dos parâmetros morfométricos mostra superioridade para o diâmetro e área de copa da castanheira-do-brasil, o que indica que essa espécie necessita de maior espaço vital e maiores espaçamentos iniciais do que as demais espécies analisadas

3. A copa da castanheira-do-brasil mostra-se menos eficiente em manter um incremento médio anual em diâmetro de $2 \mathrm{~cm}$, pois para isso necessita de uma área de copa 50\% maior do que o ipê-roxo, e 53\% maior do que o jatobá.

\section{Referências}

ASSMAN, E. Waldertargskunde. Müchen: Bayr. Landw 1961. 490p.

BURGER, H. Baumkrone und zuwachs in zwei hiebsreifen fichtenbeständen. Mitteilungen der Schweizerischen Anstalt für das Forstliche Versuchswesen, v.21, p.147-176, 1939.
CATINOT, R. Le present et l'avenir des forêts tropicales humidos. Paris: BFT, 1974. p.50.

DURLO, M.A. Relações morfométricas para Cabralea canjerana (Well.) Mart. Ciência Florestal, v.11, p.141-149, 2001.

DURLO, M.A.; DENARDI, L. Morfometria de Cabralea canjerana, em mata secundária nativa do Rio Grande do Sul. Ciência Florestal, v.8, p.55-56, 1998.

NUTTO, L. Manejo do crescimento diamétrico de Araucaria angustifolia (Bert.) O. Ktze. baseado na árvore individual. Ciência Florestal, v.11, p.9-25, 2001.

NUTTO, L.; TONINI, H.; BORSOI, G.A.; MOSCOVICH, F.A.; SPATHELF, P. Utilização dos parâmetros da copa para avaliar o espaço vital em povoamentos de Pinus elliottii Engelm. Boletim de Pesquisa Florestal, v.42, p.110-122, 2001.

SCHNEIDER, P.R. Introdução ao manejo florestal. Santa Maria: Ed. UFSM, 1993. 348p.

SPATHELF, P.; NUTTO, L.; SELING, I. Condução do crescimento em diâmetro de Eucalyptus grandis com base em árvores individuais. In: SIMPÓSIO LATINO-AMERICANO SOBRE MANEJO FLORESTAL, 1., 2000, Santa Maria. Anais. Santa Maria: UFSM, 2000. p.471-490.

STERBA, H. Using permanent sampling of individual trees as source of information for forest management. In: SIMPÓSIO LATINOAMERICANO SOBRE MANEJO FLORESTAL, 2., 2000, Santa Maria. Anais. Santa Maria: UFSM, 2001. p.19-37.

WADSWORTH, F.H. Producción florestal para America Tropical. Washington: USDA, 2000. 602p.

YARED, J.A.G.; KANASHIRO, M.; VIANA, L.M.; CASTRO, T.C.A. Comportamento silvicultural da castanheira (Berthollethia excelsa) em diversos locais na Amazônia. In: CONGRESSO FLORESTAL PANAMERICANO, 1.; CONGRESSO FLORESTAL BRASILEIRO, 7., 1993, Curitiba. [Anais]. Curitiba: SBS/SBEF, 1993. p.416-419.

Recebido em 23 de abril de 2004 e aprovado em 22 de fevereiro de 2005 